\title{
SMART-HIP - Entwicklung und Auslegung eines Sensorsystems für die Messung der Verankerung von Implantaten

\author{
Thomas Bender ${ }^{1}$, Martin Sass ${ }^{1}$, Rainer Bader ${ }^{1}$, Daniel Kluess ${ }^{1}$
} \\ ${ }^{1}$ Universitätsmedizin Rostock, Orthopädische Klinik und Poliklinik
}

\begin{abstract}
:
Loosening of total hip replacements (THR) is still the main reason for revision. Approximately 36,000 revisions are necessary in Germany per year. Due to low sensitivity of currently applied diagnostic methods, e.g. radiographs, the loosening status is often identified late. To optimize the accuracy, an acoustic method was investigated. Therefore, implants with an integrated magnetizable ball were inserted in artificial femoral bone models. The ball inside the implant was excited externally using a pair of electromagnetic coils to produce an impingement of the ball and thus perform a sound analysis. In this study, a sensor array, which is based on the vibration and attenuation analysis, is used to classify three different levels of loosening.
\end{abstract}

Key words: acoustic testing; vibration analysis, total hip replacement, aseptic loosening, intelligent implant, magnetic induction; loosening sensor

\section{Einführung}

Einen Hauptgrund für Revisionsoperationen bei Hüftendoprothesen stellt die Lockerung von Implantatkomponenten dar [1]. Rund 36.000 Revisionen sind jedes Jahr in Deutschland erforderlich [2]. Implantatlockerungen können z.B. durch abriebinduzierte Osteolysen, eine unzureichende Primärstabilität oder Spannungsabschirmung mit sukzessivem Knochenabbau hervorgerufen werden [3]. Dadurch kann es zu einem stufenweise fortschreitenden Prozess der aseptischen Lockerung kommen. Dieser reduziert die mechanische Integration zwischen Implantatoberfläche und Knochen, wobei Knochengewebe durch fibröses Gewebe ersetzt werden kann (periprothetische Membran). Aufgrund unzureichender Sensitivität der derzeit angewandten bildgebenden Diagnosemethoden, wie z.B. Röntgenbilder, FDG-PET, Arthrographie oder Szintigraphie, wird der Lockerungszustand häufig zu spät oder falsch erkannt [4]. Eine Diagnose wird meist erst nach Auftreten klinischer Symptome, Beschwerden oder Schmerzen durchgeführt. In Abbildung 1 ist ein Vergleich zwischen einem fest eingewachsenen und einem gelockerten Hüftendoprothesenstiel dargestellt. Das Röntgenbild auf der rechten Seite zeigt einen gelockertern Hüftstiel mit einem deutlichen schwarzen Saum um das Implantat (radiolucent line) [5] [6].
In vielen Fällen ist der schwarze Saum jedoch weniger deutlich sichtbar und die Lockerung kann nicht genau bestimmt werden. Aus diesem Grund erreichen diese Methoden Sensitivitäten und Empfindlichkeiten unter $100 \%$. Bei einer zu spät diagnostizierten Lockerung können massive Osteolysen (Knochenabbau) und Knochendefekte auftreten und eine weitere Revisionsoperation gestaltet sich komplexer.
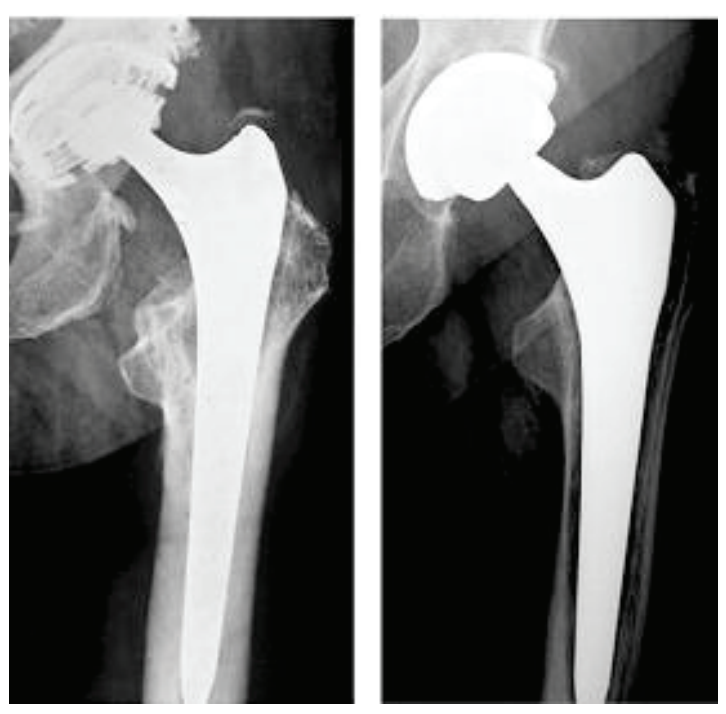

Abbildung 1: Röntgenaufnahmen eines geraden Hüftstiels. Links: Feste Verankerung im Knochen durch Osseointegration des Implantats. Rechts: Sichtbarer dunkler Saum durch ausgeprägte aseptische Lockerung. 
Um die Genauigkeit der Lockerungsdiagnose zu erhöhen, wurde eine akustische Methode entworfen [7]. Diese Diagnose hat das Potential einer regelmäßigen Untersuchung ohne Strahlenbelastung und kann einen zusätzlichen Indikator bei einer unsicheren Diagnose bieten.

\section{Methoden}

Beim akustisch-mechanischen Messprinzip wird ein Feder-Masse-Schwinger mit einer magnetisierbaren Kugel in den Hüftstiel eines künstlichen Hüftgelenks eingesetzt. Der Schwinger wird von außen durch das magnetische Wechselfeld einer elektromagnetischen Spule ausgelenkt. Dabei schlägt die Kugel an die Innenwand des Implantats an und erzeugt Vibrationen, die sich in Form von Schallwellen ausbreiten (Abbildung 2).

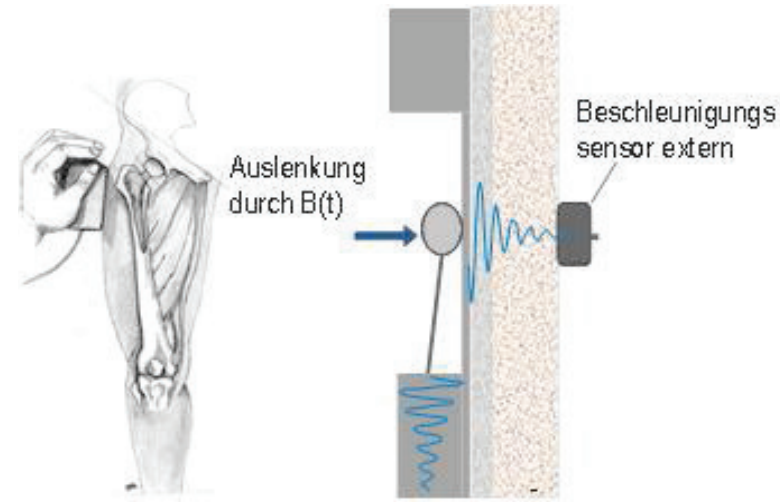

Abbildung 2: Funktionsprinzip akustischmechanische Messung

Eine Veränderung des Verankerungszustands der Hüftendoprothese hat dabei einen Einfluss auf die Schallausbreitung.

Ein Messsystem zur Aufnahme der resultierenden Vibrationen besteht im vorliegenden Testaufbau aus drei piezoelektrischen Beschleunigungsaufnehmern der Firma Metra (KS95B100, Metra, Radebeul, Deutschland). Für erste Messungen wurden diese direkt an einem künstlichen Knochenmodell aus geschlossenzelligem Polyurethanschaum (Dichte $\rho=20$ pcf, Sawbones, Vashon, USA) mit variabler Position befestigt. Um eine reproduzierbare Anbindung an den Kunstknochen zu gewährleisten, wurden Sensorbefestigungen aus Aluminiumplättchen konstruiert und in Aussparungen auf dem Kunstknochen mit einer Klebeverbindung integriert. Die Sensoren wurden mit einer Schraubverbindung an diesen Plättchen befestigt. Es wurden je fünf Sensorpositionen auf allen vier Seiten medial, lateral, anterior und posterior vorgesehen (Abbildung 3). Diese Methode gewährleistet eine reproduzierbare Befestigung der Sensoren am Messobjekt und eine sichere und gleichbleibende Signalübertragung zwischen Implantatsystem und den Sensoren.

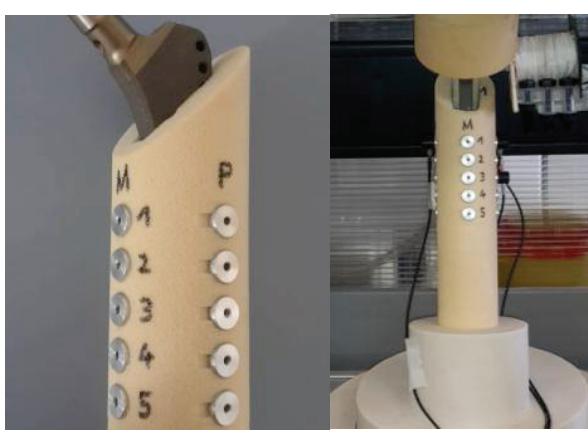

Abbildung 3: Kunstknochen mit SensorBefestigung (Links) Kunstknochen mit SensorBefestigung und Sensor im Prüfstand (rechts)

Für die Klassifizierung des Lockerungsgrads wurden drei unterschiedliche Zustände erstellt.

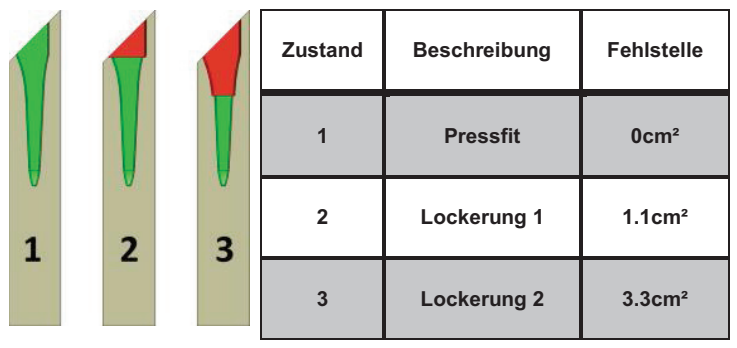

Abbildung 4: Simulierte Lockerungsgrade im Kunstknochen

Der Kunstknochen wurde mit verschiedenen Defekten im oberen, proximalen Bereich versehen. In diesen Defektbereichen gibt es keine direkte Verbindung zwischen dem Implantat und den Kunstknochen (Abbildung 4). Dieses Knochenmodell wurde in einen experimentellen Aufbau integriert. Zusätzlich ist es mit diesem Aufbau möglich, eine Gewichtskraft auf die Hüftendoprothese zu übertragen und ein Patientengewicht $\mathrm{zu}$ simulieren. Dieses Gewicht kann variabel ab einer Größe von $12 \mathrm{~kg}$ eingestellt werden. Darüber hinaus ist das System mechanisch von der Umgebung entkoppelt. Die Entkopplung an der Femur- und der Acetabulumkomponente erfolgt durch Dämpfungselemente aus einem RTV-Silikon (Abbildung 5).

Mit Hilfe eines LabView-Programms (LabVIEW 2013, National Instruments, USA) wird ein Rechtecksignal erzeugt und mit einer Messkarte NI USB6216 (National Instruments, USA) in ein analoges Signal gewandelt. Dieses Ausgangssignal im Bereich von maximal +/7.5V wird mit einer konstanten 10-fachen Verstärkung an das extrakorporale Spulenpaar angelegt. Durch das erzeugte magnetische Wechselfeld kommt es zur Auslenkung und 
dem anschließenden Anschlagen der Kugel im Implantat. Dadurch entstehen akustische Schwingungen die sich aus dem Implantat über den Kunstknochen ausbreiten. Diese Schwingungen können als Körperschall von den Beschleunigungssensoren erfasst und aufgenommen werden. Dabei wird das Signal der Sensoren mit einem Ladungsverstärker (M68D3, Metra, Radebeul, Deutschland). aufbereitet und mit einer Messkarte $\mathrm{NI}$ USB6216 digitalisiert.

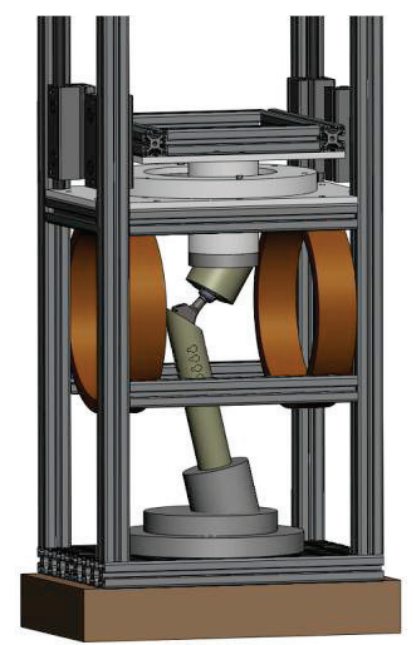

\section{Abbildung 5: Prüfstand}

Das digitale Signal im Bereich von +/- 10V wird mit Hilfe eines LabView-Programms als TDMSDatei für weitere Auswertungen abgespeichert (Abbildung 6).

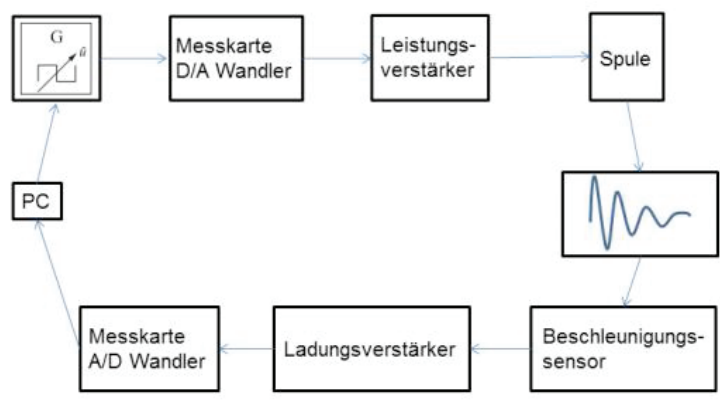

\section{Abbildung 6: Blockschaltbild der Messstrecke}

Die einzelnen Datensätze wurden mit einer Abtastfrequenz von $80 \mathrm{kHz}$ und einer Auflösung von 16 Bit über einen Zeitraum von fünf Sekunden aufgenommen. Es wird das Zeitsignal von allen drei Sensoren, das Anregungssignal und das Triggersignal aufgezeichnet. Für die aktuelle Untersuchung wurden 121 Einzelmessungen mit je 5 Anschlägen sowie das dazugehörige Abklingverhalten aufgenommen (Tabelle 1).
Tabelle 1: Übersicht der Einzelmessungen

\begin{tabular}{|c|c|c|}
\hline Zustand & Beschreibung & Messungen \\
\hline 1 & Pressfit & 46 \\
\hline 2 & Lockerung 1 & 15 \\
\hline 3 & Lockerung 2 & 60 \\
\hline $1+2+3$ & Gesamt & 121 \\
\hline
\end{tabular}

Zur Auswertung der einzelnen Anschläge werden diese einzeln in einem 10 ms Fenster ausgeschnitten und analysiert. Diese Daten können mit einer weiteren LabView-Software weiterverarbeitet werden. Dabei erfolgt die Berechnung des Frequenzspektrums des Zeitsignals mithilfe der FFT (Fast Fourier Transformation). Hierbei erlaubt die Analyse der Dämpfungseigenschaften des Zeitsignals und des Frequenzspektrums der aufgezeichneten Sensorsignale einen ersten Rückschluss auf den Grad der Lockerung des Hüftstiels (Abbildung 7).
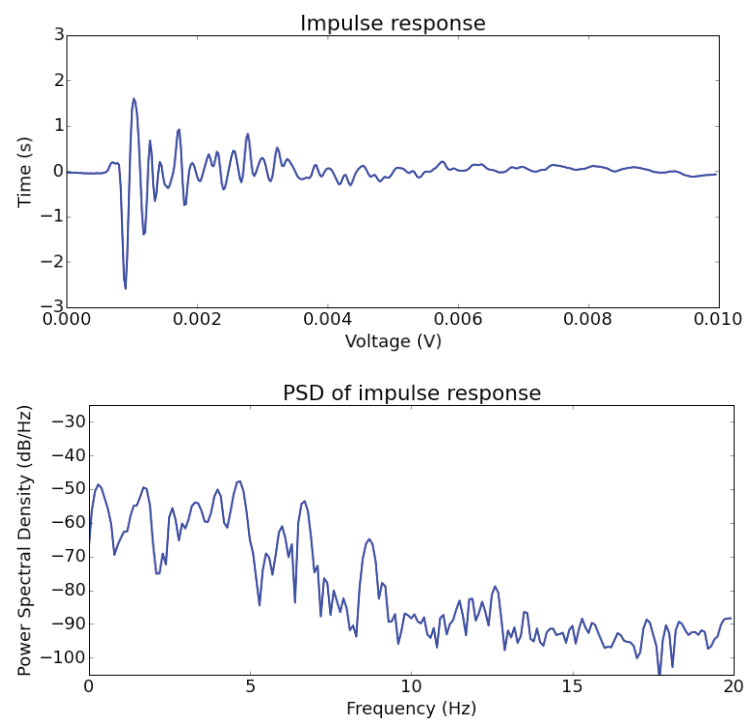

Abbildung 7: Zeitsignal und Frequenzspektrum

Die resultierenden Datensätze der FFT decken einen Frequenzbereich von $0 \mathrm{~Hz}$ bis $20 \mathrm{kHz}$ mit 201 Stützstellen ab. Diese Datensätze werden in zwei zufällig ausgewählte Untermengen aufgeteilt. Die erste Teilmenge wird als Trainingssatz und die zweite Untermenge zur Auswertung des Klassifikators benutzt. In einem ersten Schritt wird eine Hauptkomponentenanalyse (PCA) auf dem Trainingssatz durchgeführt, um die Dimensionalität der Daten von 201 Stützstellen auf drei Hauptkomponenten zu reduzieren [8]. In einem zweiten Schritt werden die transformierten Trainingsdaten verwendet, um eine Support Vector Machine (SVM) zur Klassifizierung zu trainieren [9]. Der Datensatz 
wird mit der PCA transformiert und zur Auswertung in die SVM gespeist und klassifiziert.

\section{Ergebnisse}

Basierend auf den Arbeiten am Prüfstand mit drei simulierten Lockerungszuständen zeigt sich, dass insbesondere bei den Frequenzanalysen fortgeschrittene Lockerungszustände erkannt und klassifiziert werden können, bei direkter Anbindung des Sensors an das Knochenmodell.

Eine Datenreduktion der Messdaten von jeweils 201 Frequenzpunkten auf drei Hauptkomponenten mit einer kumulierten Varianz von $96 \%$ wurde mit einer PCA (Hauptkomponentenanalyse, Principal Component Analysis) durchgeführt. Die Ergebnisse der Auswertung zeigen, dass alle Messungen korrekt von der SVM (Genauigkeit $=1,0$ und Recall $=1,0)$ vorhergesagt wurden (Abbildung 8).

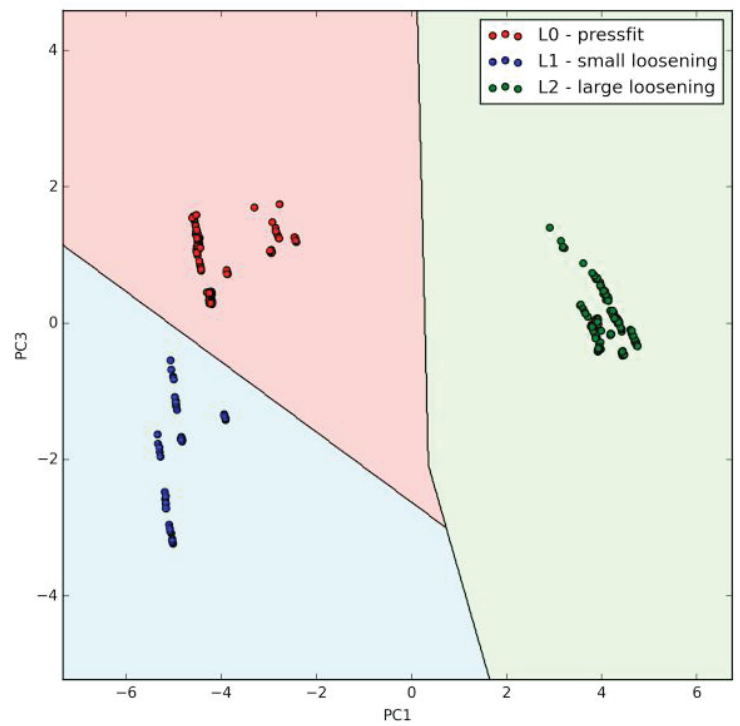

Abbildung 8: Klassifikation von 3 Lockerungszuständen mittels SVM

Die Ergebnisse bestätigen vorangegangene Arbeiten, welche in dem vorliegenden Projekt fortgeführt wurden [7].

\section{Diskussion und Ausblick}

Erste Ergebnisse der experimentellen Untersuchung von erzwungenen Schwingungen eines Hüftimplantates in einem künstlichen Knochenmodell zeigen vergleichbare Tendenzen zu mit Hilfe von numerischen Simulationen durchgeführten Analysen [10]. Aus den betrachteten Frequenzspektren lassen sich unterschiedliche Lockerungszustände identifizieren. Die Untersuchungen belegen, dass mittels der akustisch-mechanischen Messung unter Beachtung geeigneter Vorgehensweisen die Klassifizierung der Lockerung eines Hüftstieles anhand von experimentellen Messungen möglich ist.

In weiteren Anpassungen des Prüfstands wird das künstliche Knochenmodell in einem Wasserbad platziert und der Sensor an einem Hautmodell von außen appliziert. In dieser Variante wird das Wasser als Gewebeersatz für Muskel- und Fettgewebe verwendet. Eine Latexschicht dient als Begrenzung des Wasserbads und als Hautimitat, die Sensoren werden direkt auf der Latexschicht appliziert.

\section{Danksagung}

Diese Studie wurde von der Europäischen Kommission im Rahmen von \#EU-606335 SMART-HIP gefördert.

\section{Referenzen}

[1] Malchau, H. et al. The Swedish total hip replacement register. The Journal of Bone \& Joint Surgery, 84(suppl 2), S2-S20 (2002)

[2] Statistical Federal Office Germany, Fallpauschalenbezogene Krankenhausstatistik (DRG-Statistik) Operationen und Prozeduren der vollstationären Patientinnen und Patienten der Krankenhäuser. DRG-Statistik 2010

[3] Garellik G, Karrholm J, Rogmark C, Rolfson O, Herberts P. Swedish Hip Arthroplasty Register - Annual Report 2011.

[4] Temmerman, et al. (2006). Diagnostic accuracy and interobserver variability of plain radiography, subtraction arthrography, nuclear arthrography, and bone scintigraphy in the assessment of aseptic femoral component loosening. Archives of orthopaedic and trauma surgery, 126(5), 316-323.

[5] Garellik G, Karrholm J, Rogmark C, Rolfson O, Herberts P. Swedish Hip Arthroplasty Register - Annual Report 2011.

[6] Krenn V. Pathologien nach Implanatation von Endoprothesen. Endoprothetik-Leitfäden für Praktiker. 2009. Berlin, deGruyter: 15-30.

[7] Ruther C. et al. Investigation of an acousticmechanical method to detect implant loosening. Medical engineering \& physics, 35(11), 1669-1675 (2013).

[8] Yeung KY and Ruzzo WL: Bioinformatics 2001 (Volume 17, Isssue 9) : 763-774 
[9] Widodo A, Yang BS and Han T. Expert Systems with Applications 2007 (Volume 32, Issue 2) 299-312

[10] Bender T. et al. Numerical investigation of an acoustic approach for loosening diagnosis of uncemented total hip stems. Bath Biomechanics Symposium, 2014

\section{Kontakt:}

Thomas Bender, Universitätsmedizin Rostock Orthopädische Klinik und Poliklinik, Doberaner Straße 142, 18057 Rostock, E-Mail: thomas.bender@med.uni-rostock.de, Tel.: +49/381/4949334 